Cahiers de recherches médiévales

\title{
Archéologie des commanderies de l'Hôpital et du Temple en France (1977-2007)
}

\section{Damien Carraz}

\section{(2) OpenEdition \\ Journals}

Édition électronique

URL : https://journals.openedition.org/crm/5753

DOI : $10.4000 / \mathrm{crm} .5753$

ISSN : 1955-2424

Éditeur

Honoré Champion

Édition imprimée

Date de publication : 20 juin 2008

Pagination : 175-202

ISSN : $1272-9752$

Référence électronique

Damien Carraz, «Archéologie des commanderies de l'Hôpital et du Temple en France (1977-2007) », Cahiers de recherches médiévales [En ligne], 15 | 2008, mis en ligne le 20 juin 2011, consulté le 15 décembre 2022. URL : http://journals.openedition.org/crm/5753 ; DOI : https://doi.org/10.4000/crm 5753 


\title{
rin
}

\section{Archéologie des commanderies de l'Hôpital et du Temple en France (1977-2007)}

\begin{abstract}
The commanderies of the Hospital and the Temple were the object, in France, of numerous archaeological investigations these last thirty years. However, realized for the greater part within the framework of preventive excavations, these researches were rarely worn in the knowledge of the historians of the military orders or monasticism in general. It is thus a question of presenting a brief panorama by distinguishing the rural sites of the urban settlements and by favouring, rather than the monumental studies, the results of the excavations confronted with the written sources. The topography of the sites of commanderies, the general organization of the conventual and utilitarian buildings as well as the presence of defensive structures are evoked at first. The everyday life inside these built sets is then envisaged from the identification of lodging houses and possible home-made functions. The collected furniture - food rests, ceramics, pieces of armament and harness confirms the synthesis, adopted by these militarized monks, between aristocratic mentalities and regular life. The organization of the ecclesial and funeral spaces arouses finally a series of questioning on the parochial functions of certain conventual churches and on the management of cemeteries rather widely opened to the laymen and particularly to the children.

Résumé: Les commanderies de l'Hôpital et du Temple ont, en France, fait l'objet de nombreuses investigations archéologiques ces trente dernières années. Toutefois, réalisées pour la plupart dans le cadre de fouilles préventives, ces recherches ont rarement été portées à la connaissance des historiens des ordres militaires ou du monachisme en général. Il s'agit donc d'en présenter un bref panorama en distinguant les sites ruraux des implantations urbaines et en privilégiant, plutôt que les études monumentales, les résultats des fouilles confrontés aux sources écrites. La topographie des sites des commanderies, l'organisation générale des bâtiments conventuels et utilitaires ainsi que la présence de structures défensives sont évoquées dans un premier temps. La vie quotidienne à l'intérieur de ces ensembles bâtis est ensuite envisagée à partir de l'identification des logis et d'éventuelles fonctions artisanales. Le mobilier recueilli - restes alimentaires, céramiques, pièces d'armement et de harnachement - confirme la synthèse, adoptée par ces religieux militarisés, entre habitus aristocratique et vie régulière. L'organisation des espaces ecclésiaux et funéraires suscite enfin une série d'interrogations sur les fonctions paroissiales de certaines églises conventuelles et sur la gestion de zones cimétériales assez largement ouvertes aux laïcs et notamment aux enfants.
\end{abstract}

Dans sa conclusion à la $41^{\mathrm{e}}$ session de Fanjeaux, Alain Demurger soulignait que le renouvellement des connaissances sur les ordres religieux militaires viendrait très probablement de l'archéologie'. Or, il faut bien avouer que l'évident dynamisme

${ }^{1}$ A. Demurger, «Conclusion », Les ordres religieux militaires dans le Midi (XII $-X I V^{e}$ siècle), Cahiers de Fanjeaux, ${ }^{\circ}$ 41, 2006, p. 363.

Cahiers de Recherches Médiévales, 15, 2008 
de la recherche sur cette forme originale de vie régulière a bien peu pris en compte les apports des sources matérielles. Aussi, ai-je bien volontiers accepté de tenter un bilan des récentes recherches archéologiques en France dans le cadre de ce dossier spécial des Cahiers de Recherches médiévales. À partir des observations accumulées au cours de ces trois dernières décennies, je considérerai ici les établissements des ordres de l'Hôpital et du Temple, en restreignant mon attention aux phases d'occupation strictement médiévales². Toutefois, un tel exercice suppose de nombreuses limites d'ordre méthodologique.

À l'exception de deux enquêtes universitaires en cours et d'un unique programme collectif de recherche consacrés aux ordres militaires ${ }^{3}$, la quasi-totalité des investigations récentes s'inscrit dans le cadre de l'archéologie préventive ${ }^{4}$. Ce bilan ne peut donc être exhaustif, même s'il doit beaucoup aux chercheurs auprès desquels j'ai sollicité des informations sur l'actualité archéologique de leurs régions

\footnotetext{
2 Je n'ai eu connaissance que de deux sites étrangers à ces deux ordres et qui ont surtout laissé des traces d'occupation moderne, l'un de l'ordre du Saint-Sépulcre à Soudaine-la-Vinadière, en Corrèze (P. Conte, "Chronique des fouilles médiévales », Archéologie médiévale, t. 26, 1996, p. 265 et t. 31, 2001, p. 427) et l'autre des Teutoniques à Suntheim, près de Rouffach, en Alsace (P. Brunel, «Rites et pratiques funéraires dans les ordres religieux militaires : étude comparative entre l'ordre de Saint-Jean de Jérusalem et l'ordre teutonique ", Cahiers alsaciens d'archéologie, d'art et d'histoire, t. 33, 1990, p. 145-51). La chronologie adoptée est assez arbitraire puisque la plupart des sites évoqués furent occupés jusqu'aux XVII ${ }^{\mathrm{e}}$ $\mathrm{XVIII}^{\mathrm{e}} \mathrm{s}$. et que les niveaux d'époque moderne ont souvent conservé des structures plus suggestives que ceux du Moyen Âge.

${ }^{3}$ Signalons, parmi les travaux universitaires, ceux que conduit L. D’Agostino (Université de Lyon 2) sur les implantations du Temple et de l'Hôpital dans les diocèses de Clermont et de Saint-Flour et ceux qu'a entamés Y. Mattalia (Université de Toulouse 2) sur les établissements de ces mêmes ordres dans les diocèses de Cahors, Rodez et Albi aux XII ${ }^{\mathrm{e}}-\mathrm{XIII}{ }^{\mathrm{e}}$ s. Si elle ne repose pas sur une démarche strictement archéologique, la thèse de V. Bessey, Les commanderies de l'Hôpital en Picardie au temps des chevaliers de Rhodes, 1309-1522, Millau, 2005, doit être signalée pour ses apports précieux sur le patrimoine monumental des commanderies. Ces travaux individuels sont rarement relayés par de véritables enquêtes collectives et il faut donc insister sur l'intérêt exceptionnel de la recherche interdisciplinaire conduite autour du grand prieuré de l'Hôpital de Toulouse, N. Pousthomis-Dalle, «Toulouse. Programme de recherche sur le grand prieuré des Hospitaliers de Saint-Jean de Jérusalem », Archéologie du Midi médiéval, t. 19, 2001, p. 181-87 ; et F. Dieulafait (dir.), Hôtel des Chevaliers de Saint-Jean de Jérusalem, Toulouse, 2005.

${ }^{4}$ On connaît bien les obstacles que doivent affronter les archéologues responsables de ces opérations «d'urgence»: les contraintes matérielles des interventions n'autorisent que rarement l'exploration complète des sites, le temps manque pour l'exploitation des données recueillies et notamment pour la publication et il est souvent difficile d'inscrire dans des problématiques d'ensemble les résultats de fouilles dictées par les hasards du «sauvetage». Même s'il est un peu sévère, on peut d'ailleurs reprendre à notre compte le jugement formulé à propos de l'archéologie des structures hospitalières par F.-O. Touati, «Problèmes d'histoire, d'architecture et d'archéologie dans l'Europe médiévale ", Archéologie et architecture hospitalières de l'Antiquité tardive à l'aube des temps modernes, dir. F.-O. Touati, Paris, 2000, p. 10-11.
} 
respectives 5 . L'état des lieux est également sommaire car il s'appuie principalement sur des données résumées, au mieux sur quelques articles, peu de sites ayant fait l'objet d'une véritable publication ${ }^{6}$. Cette synthèse provisoire - c'est la loi du genre - n'a donc d'autre but que de souligner certaines observations récurrentes et d'énoncer ainsi d'éventuelles directions de recherche.

Rien ne caractérise particulièrement la méthodologie des recherches appliquées aux ordres militaires sauf peut-être, la richesse, parfois assez exceptionnelle, des sources écrites et iconographiques d'Ancien Régime et notamment des visites de l'ordre de Malte. Sollicités depuis longtemps, notamment pour évaluer la part des héritages médiévaux dans l'organisation topographique des commanderies, ces procès-verbaux de la fin du Moyen Âge et de l'époque moderne doivent, autant que possible, être confrontés aux observations de terrain ${ }^{7}$. Même si les visites et les plans des bâtiments des commanderies ne dévoilent qu'un état tardif remontant au mieux à la fin du Moyen Âge, leur analyse régressive apporte des données capitales, notamment en milieu urbain. Pour le Moyen Âge central, les actes de la pratique permettent de glaner quelques mentions de bâtiments assorties de repères chronologiques et l'on peut encore attendre beaucoup des inventaires de biens dressés lors de l'arrestation des Templiers puis de la dévolution de leur patrimoine à l'Hôpital ${ }^{8}$. Lorsque subsistent encore des élévations - une situation

\footnotetext{
5 Il m'est particulièrement agréable de remercier pour leur disponibilité : Jean-Marie Allard, Valérie Bessey, Annie Bolle, Laurent D’Agostino, Jean-Marc Mignon, Éric Normand, JeanPaul Nibodeau, Nuria Nin, Nelly Pousthomis-Dalle, Robert Thernot, Cécile Treffort et Robert Vinas. Michèle Bois, Emmanuelle du Bouëtiez de Kerorguen, Philippe Racinet et Philippe Rigaud n'ont pas été en mesure de me livrer des informations nouvelles, mais ont eu l'amabilité de me répondre. Les éventuelles erreurs de lecture ou d'interprétation relèvent naturellement de ma seule responsabilité.

${ }^{6}$ Par souci d'allégement, je ne répéterai pas toujours les références bibliographiques dans les notes ultérieures et signalerai simplement, dans le texte, le numéro de note de la première occurrence entre crochets.

${ }^{7}$ M. Miguet, «Problèmes de méthodologie liés aux visites des commanderies. Photographie et cartographie des vestiges ", Las ordenes militares en el Mediterraneo occidental (s. XIIXVIII), Madrid, 1989, p. 11-5. Je me suis, moi-même, fondé sur cette documentation des $\mathrm{XVI}^{\mathrm{e}}-\mathrm{XVIII}{ }^{\mathrm{e}}$ s. pour restituer l'organisation tardo-médiévale de la commanderie d'Avignon, disparue au cours du XIX ${ }^{\mathrm{e}}$ s. à l'exception de sa chapelle, D. Carraz, "Une commanderie templière et sa chapelle en Avignon: du Temple aux chevaliers de Malte », Bulletin Monumental, t. 154, 1996, p. 7-24.

${ }^{8}$ Ces inventaires peuvent aider à l'identification de certains bâtiments toujours en élévation, comme l'avait fait, pour Jalès, R. Saint-Jean, « La commanderie de Jalès : les bâtiments, XII ${ }^{\mathrm{e}}$ $\mathrm{XVIII}^{\mathrm{e}}$ siècles », Revue du Vivarais, t. 91, 1987, p. 53-70. Lorsqu'ils sont particulièrement détaillés, ces documents autorisent une véritable visite virtuelle de la maison. L'enquête effectuée à Arles en 1308 a ainsi permis de dresser un plan de l'organisation générale des espaces au sein de l'enclos templier dont on reconnaitra volontiers le caractère hypothétique, mais qui n'en est pas moins suggestif, D. Carraz, L'Ordre du Temple dans la basse vallée du Rhône (1124-1312). Ordres militaires, croisades et sociétés méridionales, Lyon, 2005, p. 266-67.
} 
souvent plus compromise en milieu urbain qu'en zone rurale ${ }^{9}$, le recours à l'archéologie du bâti permet de distinguer les états médiévaux des transformations d'époques moderne et contemporain $\mathrm{e}^{10}$. La méthode stratigraphique appliquée aux maçonneries et aux éléments de couverture permettra encore de renouveler la connaissance de l'architecture religieuse des ordres militaires ${ }^{11}$. Il faut citer à ce titre l'étude collective conduite par le Laboratoire d'Archéologie Médiévale Méditerranéenne sur l'église Saint-Jean de l'Hôpital d'Aix (pl. 1) qui a notamment montré la gestion parfaitement rationalisée de la mise en œuvre des matériaux et de l'avancée d'un chantier liée au projet de réorganisation de la nécropole comtale ${ }^{12}$. En amont des investigations effectuées sur les élévations ou dans le sol, les enquêtes programmées pourront encore tirer grand profit des campagnes de prospection, aériennes ou au sol, toujours à même de révéler des sites ou des bâtiments inconnus ${ }^{13}$.

\footnotetext{
${ }^{9}$ Pour des raisons qui tiennent autant à l'histoire propre de ces ordres qu' aux restructurations urbaines des $\mathrm{XIX}^{\mathrm{e}}-\mathrm{XX}^{\mathrm{e}}$ s., les patrimoines monumentaux sont beaucoup moins bien préservés en ville. Le cas de la commanderie du Temple de Grasse (Alpes-Maritimes) est significatif à cet égard : situé hors-les-murs de la ville, le site médiéval, dont les Capucins héritèrent en 1605, a été complètement arasé par la construction d'une usine au XIX ${ }^{\mathrm{e}}$ s., A. Dumont, «Chronique... », Archéologie médiévale, t. 27, 1997, p. 195-96.

${ }^{10}$ L'étude du « donjon » de la commanderie de Richerenches (Vaucluse) a permis de restituer la chronologie relative de la construction, les divisions internes, les espaces de circulation et les ouvertures de cet édifice, jusque là parasité par des cloisonnements post-médiévaux, J.-M. Mignon (dir.), Richerenches. Maison templière. Rapport d'étude architecturale et archéologique, SADV, 2002. Un travail semblable conduit au Fort Saint-Jean de Marseille a révélé des parties de la chapelle romane de l'Hôpital dont l'existence était jusque-là insoupçonnée, Ph. Mellinand, «Marseille. Fort Saint-Jean », Bilan scientifique de la Région Provence-Alpes-Côte d'Azur. 1993, Paris, 1994, p. 122 ; et O. Ginouvez, ibid., 1995, p. 136.

${ }^{11}$ Si les chapelles «romanes » ont d'abord attiré l'attention des historiens d'art, les dernières recherches tendent plutôt à souligner le rôle des ordres militaires dans la diffusion des nouvelles formes inspirée de l'opus francigenum. Voir par exemple C. Andrault-Schmitt, Les églises des Templiers de la Creuse et l'architecture du XIII ${ }^{e}$ siècle en Limousin, Poitiers, 1998 ; D. Carraz, "Les ordres militaires, le comte et les débuts de l'architecture gothique en Provence ", Bulletin de la Société de l'histoire et du patrimoine de l'ordre de Malte, $\mathrm{n}^{\circ} 13$, 2003, p. 45-55; et C. Balagna, "L'influence des ordres hospitaliers et militaires dans l'émergence de l'architecture gothique en Gascogne et Toulousain ", Les ordres religieux militaires dans le Midi, op. cit., p. 213-38.

12 A. Hartmann-Virnich, «Aix-en-Provence, église Saint-Jean-de-Malte : approches d'un premier chantier du gothique rayonnant en Provence », Bulletin monumental, t. 154, 1996, p. 345-50.

${ }^{13}$ M. Miguet, Templiers et Hospitaliers en Normandie, Paris, 1995, fut l'un des premiers à confronter la photographie aérienne aux sources écrites et iconographiques. Cette démarche régressive permit notamment d'éclairer la constitution des terroirs autour des commanderies et livra des observations capitales sur l'organisation monumentale de ces dernières en révélant même quelques édifices majeurs. Sur cent sites templiers et hospitaliers recensés dans les limites du diocèse de Clermont, quarante-sept ont livré suffisamment d'informations concernant des bâtiments résidentiels et/ou religieux, dix-huit édifices cultuels ont été
} 
En attendant les résultats de recherches futures, trois grandes orientations m'ont paru émerger de la trentaine d'études monographiques portées à ma connaissance. Le premier problème concerne la topographie des sites d'implantation des maisons religieuses, la présence de structures défensives et la configuration générale des bâtiments au sein de ces ensembles conventuels. La seconde approche envisage la vie quotidienne à l'intérieur de ces ensembles et notamment les problèmes posés par l'identification des fonctions résidentielles et artisanales. L'évolution des lieux de culte et la fonction funéraire attachée aux commanderies suscitent enfin la dernière série de questionnements ${ }^{14}$.

\section{Configurations générales des établissements conventuels}

\section{Les préoccupations défensives}

La situation stratégique des implantations rurales, en bordure de voies fréquentées ou de cours d'eau, a été remarquée depuis longtemps ${ }^{15}$. Les interventions archéologiques confirment la prédilection des frères guerriers pour des sites défensifs : la maison de l'Hôpital de Corbeil s'est installée sur une île de l'Essonne ${ }^{16}$, celles de La Salvetat de Serres ${ }^{17}$ ( $\mathrm{H}^{\mathrm{te}}$-Garonne) et du Temple de TargonMontarouch $^{18}$ (Gironde) ont pris position sur une motte, tandis que l'établissement templier de Carlat (Cantal) a réinvesti une position castrale attestée depuis 839 sur un plateau basaltique de 15 à $25 \mathrm{~m}$ de hauteur ${ }^{19}$. De telles configurations de terrain

repérés et une dizaine de lieux ont révélé un potentiel suffisant pour faire l'objet d'une étude du bâti, L. D’Agostino, «Prospection thématique. Les commanderies du Temple et de l'Hôpital de Saint-Jean de Jérusalem. Diocèse de Clermont », Bilan scientifique de la Région Auvergne. 2002, Paris, 2003, p. 140-42.

${ }^{14}$ Le temps et les limites éditoriales impartis à la rédaction de cette synthèse m'ont obligé à écarter des approches plus traditionnelles - comme l'évolution stylistique des constructions ou bien des thématiques dont l'ampleur réclamerait une enquête spécifique - comme l'impact des commanderies sur le peuplement rural et sur l'urbanisation. Les mêmes contraintes de rédaction m'ont encore engagé à m'en tenir à une approche franco-centrée, même si je mesure l'intérêt qu'il y aurait à confronter les résultats des fouilles métropolitaines à d'autres écoles archéologiques. Enfin, il n'a pas été possible d'amorcer ici une comparaison, qui aurait été certainement fructueuse, avec les établissements d'autres ordres réguliers.

${ }^{15}$ R. Oursel, «L'architecture religieuse des Templiers », Routes romanes, vol. 3, La garde de Dieu, La Pierre-qui-Vire, 1986, p. 352-61, a consacré au caractère stratégique des implantations templières des pages suggestives, confirmées depuis par toutes les études régionales.

${ }^{16}$ E. du Bouëtiez de Kerorguen, V. Delattre, « La commanderie Saint-Jean de l'Isle à CorbeilEssonnes. Essai d'interprétation des données archéologiques et funéraires », Archéologie médiévale, t. 32, 2002, p. 105-34.

${ }^{17} \mathrm{La}$ butte artificielle domine une terrasse alluviale de la Garonne, en bordure d'une voie romaine, G. Manière, «Un établissement des Hospitaliers de Saint-Jean de Jérusalem, La Salvetat de Serres (com. de Lavelanet de Comminges, Haute-Garonne)», Archéologie médiévale, t. 7, 1977, p. 179-227.

${ }_{18}$ B. Demotz, "Chronique... », Archéologie médiévale, t. 11, 1981, p. 308-09.

${ }^{19}$ L. D’Agostino, «Chronique... », Archéologie médiévale, t. 36, 2006, p. 273-74. 
ont donc souvent conduit les ordres à s'établir sur des sites préalablement occupés, mais presque toujours après un hiatus de plusieurs siècles: la fréquentation a pu remonter à la période carolingienne à Fontsèche ${ }^{20}$ (Charente-Maritime), au HautEmpire à Corbeil, au néolithique à Carlat... Par conséquent certains sites donnés aux ordres militaires étaient parfois déjà dotés d'un lieu de culte - Fontsèche, SainteVaubourg (Seine-Maritime), Carlat...-, mais il est rare que ces derniers aient été mis au jour.

Des dispositifs défensifs sont presque constamment attestés par les sources écrites comme par les vestiges matériels, même si l'on sait qu'ils résultent le plus souvent d'aménagements tardifs ${ }^{21}$. La commanderie du Temple du Mas Déu (Pyrénées-Orientales) était protégée par une imposante courtine à laquelle étaient adossés les bâtiments conventuels et les communs. Ce vaste quadrilatère mesurant jusqu'à $85 \mathrm{~m}$ sur son côté le plus long, et dont certains tronçons s'élèvent encore aujourd'hui jusqu'à $5 \mathrm{~m}$ de haut, comportait à l'origine quatre tours d'angle rectangulaires $^{22}$. Mais on ne sait s'il faut attribuer ces fortifications aux Templiers ou bien aux Hospitaliers. Le cas est plus net à Jalès (Ardèche) où la commanderie templière a longtemps gardé l'aspect d'une ferme ouverte jusqu'à la guerre de Cent Ans, lorsque les Hospitaliers la transformèrent progressivement en maison-forte en protégeant l'entrée par une tour et en enveloppant les bâtiments par de hautes courtines renforcées par des échauguettes [n. 8]. Au Temple-sur-Lot (Lot-etGaronne) ou à Targon-Montarouch, les fouilleurs ont également trouvé traces de courtines avec chemins de ronde entourées de fossés, mais ces structures ne sont pas antérieures aux $\mathrm{XV}^{\mathrm{e}}-\mathrm{XVI}^{\mathrm{e}}$ siècles ${ }^{23}$. La protection a toutefois pu être plus sommaire : il n'y avait à l'Hôpital de Fontsèche que des palissades doublées de simples fossés de drainage [n. 20], tandis qu'à La Salvetat de Serres, la butte était simplement fossoyée [n. 17]. Retenons de ces quelques exemples que les véritables mises en défense datent pour la plupart de la guerre de Cent $\mathrm{Ans}^{24}$.

\footnotetext{
${ }^{20}$ A. Bolle (dir.), Tonnay-Charente (17). La commanderie de Fontsèche, rapport de fouille d'archéologie préventive, INRAP, 2007.

${ }^{21} \mathrm{~J}$ 'écarte ici la question des châteaux stricto sensu dont les frères purent hériter à la suite de donations - comme les Hospitaliers à Manosque (Alpes de $\mathrm{H}^{\mathrm{te}}-\mathrm{P}^{\mathrm{ce}}$ ) - ou qu'ils élevèrent euxmêmes - comme les Templiers à La Couvertoirade (Aveyron).

22 J. Fuguet Sans, «L'arquitectura dels templers a la Catalunya Nord», Les Templiers en Pays catalan, Perpignan, 1998, p. 171-211 (ici, p. 173-83).

${ }^{23}$ Pour le Temple-sur-Lot: J. Pons, M. Daynès, "Chronique ...», Archéologie médiévale, t. 22, 1992, p. 468-69; et Targon: cf. n. 18. À Bourgneuf (Charente-Maritime), l'église templière était protégée par un profond fossé et vraisemblablement accessible par un pontlevis, É. Normand, «Chronique... », Archéologie médiévale, t. 28, 1998, p. 215-16. De tels enclos défensifs ont pu exister en milieu villageois, comme à Saint-Christol (Hérault) où le castrum s'est regroupé autour de la maison de l'Hôpital. La fouille a mis au jour un fossé dont on n'a pu déterminer s'il entourait l'ensemble du village ou bien seulement les bâtiments de la commanderie, Cl. Raynaud, Saint-Christol (Hérault). Genèse du centre villageois, rapport de fouille de sauvetage, CNRS, 2001.

${ }^{24}$ La campagne de fortification engagée par les Hospitaliers sur l'ensemble de leurs établissements du Larzac illustre bien ce contexte d'insécurité, J. Miquel, «La commanderie
} 
Le même souci de protection ressort des sites urbains, d'autant plus que les commanderies, très fréquemment implantées en périphérie des centres-villes, devinrent plus vulnérables à partir du $\mathrm{XIV}^{\mathrm{e}}$ siècle $^{25}$. Avant même les troubles de la fin du Moyen Âge, certaines maisons purent même s'intégrer au système défensif de la ville et déployer un appareil militaire, comme l'Hôpital de Marseille ou le Temple $\mathrm{d}^{\text {'Hyères }}{ }^{26}$. En ville, le besoin d'isolement indispensable à toute vie régulière a encore pu conduire, plus tôt qu'à la campagne, à la constitution d'enclos fermés ${ }^{27}$. Mais seules les sources écrites et figurées nous laissent généralement percevoir les dispositifs de défense qui sont très rarement parvenus jusqu'à nous ${ }^{28}$. Connue par l'iconographie, la courtine munie de quatre tours englobant le complexe hospitalier d'Aix a ainsi été repérée lors des fouilles conduites en $2001^{29}$. Des bâtiments furent détruits dans la deuxième moitié $\mathrm{du} \mathrm{XIV}^{\mathrm{e}}$ siècle pour laisser place à cette muraille de 2,30 à $3 \mathrm{~m}$ de large dont la construction peut être contemporaine de la fortification de l'église. En 1495 pourtant, l'église et le logis apparaissent en mauvais état et, au cours de la fin du $\mathrm{XV}^{\mathrm{e}}$ et du XVI ${ }^{\mathrm{e}}$ siècles, une partie du site est délaissée tandis que certains bâtiments sont détruits et remplacés par une large excavation qui correspondrait au négatif du rempart démantelé ou bien à un fossé défensif (ill. 1). Les textes permettraient de multiplier de tels exemples pour l'ensemble des régions qui souffrirent du contexte belliqueux des $\mathrm{XIV}^{\mathrm{e}}-\mathrm{XV}^{\mathrm{e}}$ siècles puis, parfois, des guerres civiles et religieuses du $\mathrm{XVI}^{\mathrm{e}}$ siècle. Les établissements ruraux furent souvent touchés plus précocement par les destructions. À La Salvetat, les fouilleurs ont identifié un violent incendie (vers 1350 ?) qui semble avoir précipité la désertion du site avant une réoccupation sous une forme plus fruste [n. 17]. Au Temple-sur-Lot, un vaste bâtiment comportant la chapelle et remontant à la fin du $\mathrm{XII}^{\mathrm{e}}$ siècle affronta

de Sainte-Eulalie. La construction des enceintes fortifiées du XV siècle », Les Hospitaliers du XII $I^{e}$ au XVII siècle, Provence historique, t. 45, 1995, p. 157-70.

${ }^{25}$ Parmi les sites archéologiques recensés, huit se situent en zone périurbaine et ce sont tous des sites hospitaliers à l'exception du Temple de Grasse : Soultz ( $\left.\mathrm{H}^{\mathrm{t}}-\mathrm{Rhin}\right)$, Épinal (Vosges, cf. A. Masquilier, "Chronique... », Archéologie médiévale, t. 31, 2001, p. 402), Le Puy, La Rochelle, Bajoles, près de Perpignan, Trinquetaille (faubourg d'Arles) et Aix-en-Provence. Deux commanderies sises en plein cœur de l'espace urbain ont en outre suscité d'importantes investigations : le Temple de La Rochelle et l'Hôpital de Toulouse.

${ }^{26}$ A. Hesnard, Ph. Bernardi, C. Maurel, «Le port», Marseille. Trames et paysages urbains, de Gyptis au roi René, Aix-en-Provence, 2001, p. 168-73; et M. Vecchione, «Un édifice templier en Provence : la tour Saint-Blaise d'Hyères », Provence historique, t. 40, 1990, p. 5775 .

${ }^{27}$ Sur l'isolement et l'enfermement, au demeurant assez relatif, des commanderies au sein de l'espace urbain, voir les situations offertes à Avignon et à Arles, D. Carraz, L'Ordre du Temple..., op. cit., p. 272-76.

${ }^{28}$ L'exemple de la commanderie Saint-Jean de la Chevalerie, placée aux avant-postes de la ville du Puy et fortifié dès 1383, est bien attesté, P.-É. Poble, «L'ordre de Saint-Jean de Jérusalem en Velay avant $1312 »$, Histoire et archéologie de l'ordre militaire des Hospitaliers de Saint-Jean de Jérusalem, Saint-Étienne, 2004, p. 154-57.

${ }_{29}$ N. Nin, "Aix-en-Provence. Musée Granet», Bilan scientifique de la Région ProvenceAlpes-Côte d'Azur. 2001, Paris, 2002, p. 95-8; et ead., «La commanderie de Saint-Jean de Jérusalem d'Aix-en-Provence », Histoire et archéologie..., ibid., p. 213-58 (ici p. 222). 
également le feu au $\mathrm{XV}^{\mathrm{e}}$ siècle. Mais cette fois-ci, l'accident ne compromit pas les reconstructions [n. 23]. La plupart des abandons furent toutefois provoqués par les conditions économiques, comme au Ruou (Var) où les restructurations administratives furent fatales à l'ancienne installation templière ${ }^{30}$.

\section{L'agencement des bâtiments}

À l'intérieur de ces enclos le plus souvent quadrangulaires, parfois également triangulaires comme à Bajoles ${ }^{31}$, et dont les surfaces purent être variables, quels sont les grands traits de l'organisation spatiale? L'emprise souvent limitée des fouilles peut entraver la perception de la disposition des bâtiments et compromettre l'interprétation d'ensemble des sites. La confrontation des données iconographiques et des observations tirées des ensembles monumentaux convenablement conservés permet toutefois d'avoir une idée satisfaisante des lieux de vie des frères. À la campagne, les importants centres domaniaux s'organisent autour de deux cours: c'est le cas à Jalès, Vaour (Tarn), Ruou (pl.2), Corbeil-Essonnes, comme en Auvergne $^{32}$. Cette différenciation des espaces répond à la double vocation, économique et résidentielle, des commanderies. Les bâtiments dédiés à l'exploitation agricole se développent autour de la basse-cour, tandis que les parties réservées à la vie conventuelle se referment autour d'un espace central évoquant assez le cloître monastique. On peine encore à cerner l'évolution chronologique de ces plans. L'achèvement de la constitution des temporels et l'enrichissement des seigneuries entraînent l'adjonction de nouveaux bâtiments aux plans primitifs. Au Ruou, l'étude archéologique a attribué à la première phase correspondant probablement au $\mathrm{XII}^{\mathrm{e}}$ siècle, la chapelle entourée du cimetière, un petit édifice turriforme (F) et un bâtiment agricole (D), auxquels il faudrait ajouter une aile résidentielle non identifiée (pl.2). Au cours du XIII ${ }^{\mathrm{e}}$ siècle apparaissent un long édifice (C), une autre construction turriforme (G1), peut-être déjà liée à une enceinte, tandis que s'établit contre la chapelle un bâtiment muni d'archères (B) qui pourrait correspondre au logis. La troisième phase, qui coïncide avec l'apogée de la commanderie, voit l'ensemble s'agrandir de trois nouveaux bâtiments $(\mathrm{E}, \mathrm{H}, \mathrm{I})$ aux fonctions non déterminées [n. 30]. Les transformations les moins malaisées à saisir en fouille concernent toutefois les réorganisations matérielles presque partout mises en œuvre après les troubles de la guerre de Cent $\mathrm{Ans}^{33}$. À Fontsèche, plusieurs

\footnotetext{
${ }^{30}$ R. Thernot, "Villecroze. Commanderie des Templiers du Ruou », Bilan scientifique de la Région Provence-Alpes-Côte d'Azur. 2004, Paris, 2005, p. 231-33.

${ }^{31} \mathrm{Cf}$. la restitution axonométrique dans J. Fuguet Sans, Templers i hospitalers, IV. Guia de la Catalunya vella, Barcelone, 2005, p. 47-9; et R. Marichal, "Chronique...», Archéologie médiévale, t. 28, 1999, p. 229-31.

32 On trouve deux cours dans les établissements hospitaliers du Mayet-d'École (Allier) et de La Racherie (Allier) et jusqu'à trois cours à La Tourette (Puy-de-Dôme) ou à Charbonnier (Puy-de-Dôme), L. D’Agostino, «Les commanderies des templiers et des hospitaliers en Auvergne : éléments pour une architecture ", Histoire et archéologie..., op. cit., p. 75-101 (ici, p. 86-9).

${ }^{33}$ Cette phase de restauration a été remarquablement analysée, à partir des sources écrites, pour les commanderies picardes, V. Bessey, Les commanderies..., op. cit..
} 
bâtiments - à usage agricole ? - furent ainsi reconstruits au $X V^{\mathrm{e}}$ siècle avec des parements assez soignés (ill. 2) [n. 20].

En ville, les travaux de réfection et d'agrandissement sont plus difficiles à appréhender: les textes ne livrent que des mentions éparses et les superficies fouillées sont souvent plus réduites. À fin du XIII ${ }^{\mathrm{e}}$ ou au début du XIV siècle, les Hospitaliers d'Aix ajoutèrent trois nouvelles pièces $(\mathrm{E}, \mathrm{D}, \mathrm{C})$ à une aile préexistante (A, pl. 1) [n. 29], tandis que ceux de Toulouse agrandirent leur cloître au XIV ${ }^{\mathrm{e}}$ siècle, tout en édifiant probablement la tour des archives et la chapelle Saint-Nicolas ${ }^{34}$ (pl. 3). La pression sur le sol urbain a conduit à des plans plus ramassés, organisés dans la plupart des cas autour d'une seule cour centrale, même si les enclos abritèrent à l'occasion jardins et annexes agricoles. À l'origine, les frères se contentèrent parfois d'aménager un édifice préexistant dont ils avaient hérité- maison, tour, fortification...-, mais que l'archéologie a peu de chances de retrouver $^{35}$. En revanche, la construction d'ensembles conventuels ex nihilo, sur des terrains vierges, se prêta probablement à des expérimentations nouvelles dont il reste encore à déterminer les influences: enclos urbains développés en Terre sainte ou bien modèles palatiaux ou monastiques?

Mentionnés par les sources écrites, les bâtiments de la vie communeréfectoire, dortoir, éventuellement salle capitulaire et parloir - et les communs cuisine, celliers... - se laissent difficilement identifier sur le terrain. Les véritables cloîtres, jusque là attestés dans des établissements exceptionnels comme le Temple et l'Hôpital de Paris ou le couvent des Hospitalières de Beaulieu (Corrèze), pourraient se révéler plus courants à la lumière des investigations en cours ${ }^{36}$. L'exploration de sites importants, comme le grand prieuré de Toulouse ou la maison de Corbeil-Essonnes, siège du prieuré de France de 1275 à 1353, a en effet révélé de tels aménagements dont on ne sait s'ils furent toujours prévus dès l'origine. Il reste à réfléchir sur la fonctionnalité de tels espaces : lieux d'inhumation selon un modèle répandu, les cloîtres étaient-ils également investis d'une dimension liturgique chez les frères guerriers? Si l'on retrouve une évidente filiation avec les plans monastiques, les recherches doivent être poursuivies afin de vérifier l'existence d'un "plan type » d'organisation des bâtiments de la vie commune. Les chapelles sontelles toujours à proximité des logis, comme au Temple de Perpignan où, d'après les visites modernes, l'église communiquait avec le «palais » au niveau de la sacristie et du chœur $^{37}$ ? Pourra-t-on déterminer l'organisation des espaces dédiés à la

\footnotetext{
${ }^{34}$ N. Pousthomis-Dalle, «Histoire et archéologie de la commanderie-grand prieuré des Hospitaliers de Saint-Jean à Toulouse : état de la recherche », Les ordres religieux militaires dans le Midi, op. cit., p. 239-64 (ici, p. 244).

35 Je me permets de renvoyer à des exemples de basse Provence attestés par les textes, D. Carraz, L'Ordre du Temple..., op. cit., p. 264-65.

${ }^{36} \mathrm{Il}$ reste à identifier ce type de structure dans des sites ruraux de moindre importance. Il n'est pas assuré, par exemple, que la galerie qui borde au sud la chapelle de l'Hôpital de Montbrison (Loire) se prolongeait sur d'autres ailes, L. D'Agostino, "Chronique... », Archéologie médiévale, t. 36, 2006, p. 283.

37 J. Fuguet Sans, «L'arquitectura dels templers... », op. cit., p. 191-92. Il semble toutefois qu'à la fin du Moyen Âge, la communication entre la chapelle et le logis ait été réservée aux dignitaires. L'accès direct entre la chambre du commandeur et la chapelle, par l'intermédiaire
} 
préparation et à la consommation des repas que des textes tardifs ne font que suggérer, à l'instar du « tinel viel » et du « grand tinel » proche d'une cuisine, situés au $\mathrm{XV}^{\mathrm{e}}$ siècle à l'Hôpital de Toulouse [n. 34, p. 245] ? Les salles capitulaires lorsqu'elles existent - ouvrent-elles nécessairement sur la $\operatorname{cour}^{38}$ ? etc.... Pour l'heure, arrêtons nous seulement à la fonction résidentielle.

\section{Aspects de la vie quotidienne à l'intérieur des commanderies}

\section{Les espaces résidentiels}

Si les logis ont mieux traversé les siècles que les parties non nobles, il faut pourtant se résoudre à n'avoir des espaces résidentiels qu'une appréciation tardive. Les dégagements d'ampleur raisonnable ont souvent mis au jour un ou plusieurs bâtiments assez vastes, mais il est significatif que les responsables d'opération renoncent la plupart du temps à interpréter de telles structures. Que penser par exemple du grand bâtiment rectangulaire divisé en deux pièces et comportant un étage dégagé à La Salvetat [n. 17] ? Ou encore de l'édifice voûté comprenant plusieurs pièces à Corbeil ${ }^{39}$ ? Il peut être tentant d'interpréter certains espaces ouverts sur la rue comme des lieux d'accueil, mais, en l'absence de textes, on voit bien le caractère hasardeux de telles suppositions ${ }^{40}$. La contribution de l'archéologie à l'étude de la fonction d'assistance des ordres militaires s'avère donc encore décevante. Il est d'autant plus délicat d'identifier en fouille des espaces proprement résidentiels que ceux-ci furent souvent aménagés à l'étage. C'est ce qu'atteste l'étude menée à Richerenches dont le premier état du bâtiment est probablement

d'une tribune, est un dispositif relativement courant, attesté par exemple à Celles (Cantal), Avignon ou Saint-Étienne de Renneville.

${ }^{38}$ La présence d'une salle capitulaire à la commanderie du Temple de Coulommiers (Seineet-Marne; H. Baptiste, La commanderie des Templiers de Coulommiers. Vie et résurrection, Paris, 2000) et au couvent des Hospitalières de Beaulieu (Corrèze) est assez rare pour être signalée. Une telle salle existait également au XIII ${ }^{\mathrm{e}} \mathrm{s}$. à la commanderie templière d'Epailly (Côte-d'Or) mais elle fut rapidement détruite pour laisser place à une chapelle élevée sur le flanc droit de l'église conventuelle, J.-B. de Vaivre, La commanderie d'Épailly et sa chapelle templière durant la période médiévale, Paris, 2005, p. 106-08.

${ }^{39}$ Une pièce d'environ $30 \mathrm{~m}^{2}$ située sur l'aile orientale du cloître est considérée comme la sacristie ou la salle capitulaire, E. du Bouëtiez de Kerorguen, "Chronique... », Archéologie médiévale, t. 30, 2000, p. 247.

${ }^{40}$ L'Hôpital d'Aix comprenait, au sud de l'église et à proximité de la rue Saint-Jean, une grande salle rectangulaire dallée datée de la deuxième moitié du XIII ${ }^{\mathrm{e}} \mathrm{s}$. (A, $\left.p l .1\right)$, tandis qu'un second bâtiment parallèle a juste été observé [n. 29]. La récente fouille de l'Hôpital Saint-Jean-du-Perrot de La Rochelle a mis au jour une vaste construction édifiée entre la fin du XII ${ }^{\mathrm{e}}$ s. et le milieu du XIII ${ }^{\mathrm{e}}$ s. de $32 \mathrm{~m}$ sur 7-10 m. Sa fonction n'a pu être établie et de nombreuses hypothèses peuvent être avancées (salle d'hôpital, cellier, entrepôt ...), tandis que son appartenance même à la commanderie n'a pas été établie avec certitude (communication personnelle de J.-P. Nibodeau, responsable d'opération). 
antérieur au milieu du XII ${ }^{\mathrm{e}}$ siècle $^{41}$. Située à l'étage, au-dessus d'un espace domestique peut-être dédié à la stabulation, la partie noble est scindée en deux par un mur de refend: la pièce la plus vaste - $21 \mathrm{~m} \mathrm{x} \mathrm{4,20} \mathrm{m-pourrait} \mathrm{bien} \mathrm{avoir} \mathrm{fait}$ office d'aula, tandis qu'une pièce orientale plus modeste - 6,50 m x 4,20 m-, dotée d'une cheminée et ornée de deux grandes arcatures aveugles, serait volontiers interprétée comme la camera du commandeur (ill. 3). Il est difficile de situer cet édifice, aujourd'hui isolé, au sein d'un ensemble monumental: une autre aile résidentielle existait nécessairement pour abriter la communauté d'une quinzaine de frères que la commanderie accueillait au XII ${ }^{\mathrm{e}}$ siècle, au temps de son plus grand développement. Mais quelle forme affectait cette résidence?

L'agrégation en un même édifice de la chapelle et du logis facilite parfois l'identification des fonctions. Ce dispositif paraît avoir été fréquemment adopté, puisqu'on le retrouve du $\mathrm{XII}^{\mathrm{e}}$ siècle - le Temple-sur-Lot comporte un long bâtiment avec chapelle en partie sud - à la fin du Moyen Âge - à Chauliac (com. Le Broc, Puy-de-Dôme) vers 1450 , le corps de logis vient englober la chapelle ${ }^{42}$. Il est fréquent que ces ailes résidentielles arborent un appareil défensif. À Richerenches, les maçonneries, déjà percées de meurtrières, furent surélevées au cours du XIII siècle et doublées d'arcs sur contreforts supportant probablement un chemin de ronde. Ces logis peuvent être dotés d'une tour, lorsqu'ils ne s'inscrivent pas euxmêmes dans un édifice turriforme comme à Chaynat (Puy-de-Dôme) [n. 42]. Les observations du bâti devront encore permettre de restituer les éléments externes disparus, tels que les hourdages, ainsi que les systèmes de circulation, comme les galeries externes en bois repérées à Celles.

$\mathrm{Si}$ les règles monastiques insistent sur la vie commune et cherchent bien à préserver les frères des tumultes du siècle, ces derniers ont pu, au fil du temps, avoir de ces préceptes une interprétation assez lâche ${ }^{43}$. La visite du Temple d'Arles effectuée en 1308 montre une subdivision poussée des espaces internes puisque l'aile résidentielle comprenait assez de pièces pour héberger la petite dizaine de frères que comptait encore la commanderie ainsi que les hôtes [n. 8]. Depuis le XIII siècle au moins, les parties nobles adoptent donc certaines caractéristiques des résidences aristocratiques dont témoignent par exemple les mentions de « salle» ou d'aula ${ }^{44}$. On ne s'étonne donc pas d'y trouver une sensibilité aux aménagements de confort et aux décors sculptés ou peints attestés surtout pour la fin du Moyen Âge -

\footnotetext{
41 J.-M. Mignon, D. Carraz, «Le donjon de la commanderie de Richerenches (Vaucluse) : premiers résultats de l'étude architecturale et archéologique », Archéologie du Midi médiéval, à paraître.

${ }^{42}$ L. D’Agostino, «Les commanderies... », op. cit., p. 92-3 ; et id., «Les moines-soldats en Auvergne et Velay », Histoire et images médiévales, $\mathrm{n}^{\circ}$ 13, 2007, p. 62-3.

43 Il n'y a pas beaucoup à attendre des textes normatifs qui, s'ils mentionnent certains espaces, se préoccupent assez peu des aspects matériels de la vie des communautés templières et hospitalières en Occident.

44 L. D’Agostino, «Les commanderies...», op. cit., p. 83; et D. Carraz, L'Ordre du Temple..., op. cit., p. 224.
} 
un champ d'investigation encore largement ouvert et susceptible de révéler d'agréables surprises ${ }^{45}$.

\section{Les structures économiques}

Maisons religieuses, les établissements des ordres militaires sont également des centres de conservation des ressources agricoles et de production artisanale. L'archéologie permet notamment d'appréhender cette dernière activité qui échappe souvent aux sources écrites. Signalons à ce titre la présence d'au moins deux fours à pain et d'un autre four remplis de déchets de fonte du bronze à La Salvetat [n. 17], comme la récente mise au jour, à Fontsèche, d'un four à pain et surtout de nombreux éléments - fosses, battitures, scories et outillages de fer - probablement liés à une activité de tannerie ou de forge ayant fonctionné entre la deuxième moitié du XIII et le $X V^{\mathrm{e}}$ siècles (ill. 4) [n. 20]. Toutefois, il est souvent difficile de déterminer les liens entre ces activités et les commanderies : ces « ateliers » relevaient-ils directement de la communauté régulière ou bien plutôt de familiers ou de tenanciers? Au grand prieuré de Toulouse, des ouvroirs étaient situés au rez-de-chaussée d'une aile de l'enclos et ouvraient sur la rue de la Dalbade, tandis que la présence d'artisans dans l'environnement des frères est remarquablement illustrée par la découverte de la charte lapidaire du cordonnier Pierre Nègre (1216) scellée sur un contrefort de l'église [n. 34, p. 242-3]. À Fontsèche, la zone artisanale sise près d'un cours d'eau ne semble pas inscrite dans l'emprise de la commanderie et devait plutôt relever du faubourg voisin. Il n'en est pas moins intéressant de noter que les milices ont suscité des activités de transformation, qu'elles aient été destinées à leurs besoins propres ou à alimenter d'éventuels circuits commerciaux.

L'évocation des structures artisanales permet également de soulever la question des aménagements hydrauliques qui, comme à Fontsèche, ont pu leur être liés. La présence de puits - qui peuvent parfois être nombreux, comme à Bajoles où l'on en a repéré au moins cinq - est une constante qui n'étonne guère. Il faudrait toutefois considérer l'ensemble des procédés de récupération et de stockage des eaux. L'action des ordres militaires dans l'aménagement des zones humides a parfois été relevée. Mais ceux-ci commencèrent probablement par s'assurer de la salubrité du site de leur maison. Dès la première phase d'installation, la source traversant le site du Ruou fut ainsi soigneusement canalisée par un conduit voûté (pl. 2) [n. 30]. Enfin, même si ces constructions emblématiques de l'essor médiéval ont suscité quelques publications concernant les ordres militaires, il y aurait encore beaucoup à écrire sur les moulins dont les commanderies ont partout et activement équipé les campagnes ${ }^{46}$. Les sources écrites permettent fréquemment de suivre sur une longue durée le devenir de ces outils de production coûteux. Mais leur recensement, par exemple dans le cadre d'enquêtes sur les mises en valeur agraires, ainsi que l'analyse archéologique des structures en élévation restent à entreprendre avant que les derniers témoins ne finissent de disparaître complètement.

\footnotetext{
45 Comme les scènes de chasse peintes vers 1450 dans la chambre du commandeur de Chaynat, L. D’Agostino, ibid., p. 84.

46 Pour une première approche du sujet, je me permets de renvoyer à D. Carraz, "Hydraulique », Dictionnaire des ordres militaires au Moyen Age, dir. Ph. Josserand, N. Bériou, Paris, Fayard, à paraître.
} 
De fait, l'agriculture et l'élevage sont bien au centre de l'économie des seigneuries de l'Hôpital et du Temple ${ }^{47}$. Ces activités nécessitent la présence de bâtiments d'exploitation, certes attestés par les textes, mais que l'archéologie a souvent du mal à appréhender lorsqu'ils n'ont pas été suffisamment conservés : granges, celliers, bergeries, étables, écuries, pigeonniers... L'attention portée aux granges héritées du Temple et de l'Hôpital en Normandie devrait inciter à multiplier les inventaires régionaux de ces aménagements particulièrement exposés aux destructions volontaires [n. 13]. Toutes les commanderies ont comporté de tels bâtiments agricoles, parfois encore fort bien conservés - les celliers entièrement maçonnés du Ruou ou de Jalès par exemple - mais pas toujours suffisamment valorisés. L'usage de structures de conservation moins coûteuses est encore couramment attesté par l'archéologie: lorsqu'ils n'ont pas été vidés par quelque fouilleur clandestin en quête de trésor templier, les silos, classiquement réutilisés comme dépotoirs, livrent bien souvent un abondant matériel faunique et céramique ${ }^{48}$.

\section{Les modes de vie}

Le mobilier recueilli principalement dans les fosses dépotoirs et dans les couches de destruction - la conservation des niveaux d'occupation étant souvent compromise par les restructurations d'époque moderne - permet de toucher certains aspects de la vie quotidienne des commanderies. Les régimes alimentaires constituent des marqueurs des différences sociales au sein des communautés religieuses élargies aux laïcs de leur entourage. À Fontsèche, l'étude archéozoologique a révélé une alimentation de type rural caractérisé par une prédominance du bœuf et la consommation d'animaux assez âgés. Ce régime lié à un niveau de vie assez moyen pourrait être celui d'une familia d'artisans [n. 20]. Les silos de Bajoles quant à eux, ont livré d'abondants restes végétaux (blé, féveroles, pois...) qui laissent supposer une alimentation essentiellement végétarienne, probablement composée de bouillies de céréales [n. 48]. Ce type d'observation doit naturellement être confronté aux analyses anthropobiologiques des populations inhumées dans les commanderies. L'étude des défunts reposant dans le cloître SaintJean de Corbeil a révélé une communauté privilégiée, épargnée par les activités physiques pénibles et préservée par les pathologies les plus courantes [n. 16]. Les individus inhumés dans le cloître de l'Hôpital de Toulouse, dans un contexte que la céramique attribue aux $\mathrm{XIV}^{\mathrm{e}}-\mathrm{XV}^{\mathrm{e}}$ siècles, étaient peu exposés au stress, aux carences alimentaires, aux fractures ou aux infections. En revanche, les atteintes dégénératives de type arthritique, souvent corrélées à l'âge, étaient fréquentes. Ce profil suggère donc une population favorisée et âgée, dotée d'une espérance de vie relativement élevée - autour de 40 ans [n. 34, p. 258]. Cet état sanitaire est sans doute

\footnotetext{
${ }^{47}$ Cette caractéristique est appelée à être nuancée en ville, où dans un contexte d'économie fortement monétarisée, les ordres militaires purent être uniquement des rentiers du sol ou des intermédiaires dans le cadre d'opérations commerciales. Mais on manque d'études approfondies sur l'intégration de ces ordres dans les circuits économiques du monde urbain. ${ }^{48}$ De telles structures ont été fouillées au Temple-sur-Lot [n. 23], à l'Hôpital d'Aix ( pl. l) [n. 29], à Saint-Christol [n. 23] et surtout à Bajoles où une aire d'ensilage a livré trente cinq fosses, P. Alessandri, «Perpignan : la commanderie hospitalière de Bajoles. Premiers éléments de la recherche », Archéologie du Midi médiéval, t. 11, 1993, p. 234-43 (ici, p. 237-41).
} 
redevable à un équilibre nutritionnel satisfaisant, mais il reste encore beaucoup à découvrir des habitudes alimentaires de ces communautés soumises à un régime moins strict que celui des ordres monastiques traditionnels.

À Bajoles, la sobriété du régime alimentaire attribué aux frères contraste avec la qualité de la vaisselle de table. En règle générale, le mobilier céramique essentiellement composé de productions communes régionales ne dénote pourtant aucun luxe inconsidéré ${ }^{49}$. On est même déçu de voir qu'il reflète très peu l'ouverture des ordres à des horizons géographiques plus lointains, comme l'Orient latin ou la Péninsule ibérique. Les céramiques glaçurées à décors vert et brun de type valencien découvertes à Aix ou à Bajoles, dans des contextes stratigraphiques des mi-XIII ${ }^{\mathrm{e}}$ $\mathrm{XV}^{\mathrm{e}}$ siècles, n'ont rien d'exceptionnel, les importations de cette région d'Espagne étant alors courantes dans l'ensemble du Midi ${ }^{50}$. La présence d'une pièce de Ceylan parmi le lot de soixante-huit monnaies tirées de la fouille du Temple de La Rochelle apparaît déjà plus singulière ${ }^{51}$. On aimerait que de telles découvertes se multiplient et permettent ainsi de valider l'impression, laissée par les sources écrites, d'institutions intégrées dans les circuits monétaires et commerciaux internationaux. L'attestation plus fréquente de pièces de cavalerie et d'armement vient heureusement conforter l'image de ces religieux militarisés. La multiplication des mentions de mors, de fers d'équidés et autre matériel de harnachement (Targon-Montarouch, Fontsèche, La Salvetat...), d'éperons (Bajoles, Fontsèche), de pointes de lance et d'armes de trait (La Salvetat, Fontsèche) ou de boucles de ceintures (Fontsèche, Corbeil, Le Mayet...) permettra sans doute de nuancer la pauvreté des armements recensés par les inventaires de la fin du Moyen Âge.

\section{Fonctions cultuelles et funéraires}

\section{Les chapelles conventuelles}

Parmi les édifices conventuels, les chapelles sont de loin les mieux documentées par les textes: leur construction ou leur utilisation ont souvent occasionné des litiges avec les autres institutions ecclésiastiques et les visites médiévales et modernes leur consacrent une attention particulière. Elles furent également un peu mieux épargnées par les destructions. L'étude des lieux de culte

${ }^{49}$ On ne saurait négliger de confronter le matériel recueilli en fouille avec les inventaires mobiliers du XIV ${ }^{\mathrm{e}}$ s. dans lesquels on trouve parfois des états détaillés des ustensiles culinaires ou des mentions d'outillages divers. Voir par exemple J. Delmas, «L'inventaire des biens de la commanderie de Sainte-Eulalie du Larzac en $1308 »$, La Commanderie, institution des ordres militaires dans l'Occident médiéval, dir. A. Luttrell, L. Pressouyre, Paris, 2002, p. 319-26.

N. Nin, «La commanderie de Saint-Jean...», op. cit., p. 220; et R. Marichal, "Chronique...», Archéologie médiévale, t. 21, 1991, p. 323-24. P. Alessandri, "Perpignan...», op. cit., p. 241, associe toutefois la présence à Bajoles de «céramique décorée de blasons d'origines géographiques très diverses » à l'accueil «des représentants d'une chevalerie se trouvant sans doute éloignée de ses terres ».

${ }^{51}$ Figuraient également une monnaie espagnole et une monnaie portugaise, J.-C. Bonnin, «Les Templiers et la mer: l'exemple de La Rochelle», La Commanderie, institution des ordres..., op. cit., p. 313-14. 
focalisa pendant longtemps l'intérêt des historiens d'art au point d'être totalement dissociés de leur environnement monumental et topographique. Les schémas établis par ces travaux devanciers restent toutefois valides : on retrouve dans les édifices bâtis pour les Templiers et les Hospitaliers des parti pris de simplicité qui se lisent notamment dans les plans - souvent à vaisseau unique et chevet plat -, les structures des élévations et l'usage modéré des décors sculptés. Cet esprit général fut naturellement perméable aux traditions régionales comme à la diffusion de nouveaux principes techniques et esthétiques. Il revient désormais à l'archéologie de dépasser l'image statique que l'on conserve souvent de ces monuments en s'attachant notamment aux phases de constructions et aux techniques de mise en œuvre. Les chapelles reflètent au mieux le niveau de richesse matérielle, le rayonnement spirituel comme les prétentions temporelles de ces communautés religieuses. On peut en effet lier l'érection d'une chapelle au cœur d'une cité à des revendications spirituelles (Hôpital d'Avignon), expliquer l'extension d'un édifice préexistant par la prospérité matérielle (Temple de La Rochelle), ou bien donner un sens politique à l'adoption d'une nouvelle esthétique architecturale (Hôpital d'Aix et Temple d'Avignon).

À l'avenir il faudra distinguer plus attentivement milieux ruraux et milieux urbains car les fonctions attachées aux lieux de culte ont souvent été différentes, tout comme les contextes des remaniements architecturaux. Les commanderies urbaines ont pu manifester un dynamisme édilitaire plus précoce. Les quelques exemples réunis ici semblent indiquer que l'on se préoccupa souvent, dès le XIII siècle, d'agrandir ou de rebâtir entièrement les chapelles urbaines. Autour de 1250, les Templiers de La Rochelle démolirent le chevet de leur chapelle romane - elle-même reconstruite vers 1140-1170 à la place d'un édifice antérieur - pour bâtir un vaste chœur voûté d'ogives ${ }^{52}$. Les Hospitaliers d'Aix érigèrent quant à eux la première église gothique de Provence entre 1272 et 1278 (pl. 1) [n. 12]. Au cours du XIV siècle, ceux de Soultz remplacèrent une chapelle à chevet plat attestée en 1234 par un édifice beaucoup plus vaste à chevet polygonal auquel seront ajoutés deux bas côtés aux $\mathrm{XV}^{\mathrm{e}}-\mathrm{XVI}^{\mathrm{e}}$ siècles $^{53}$. Les dimensions des chapelles, qui ne reflètent pas toujours l'importance réelle des communautés, invitent à réfléchir sur le sens spirituel ou symbolique - de tels investissements financiers à priori éloignés des missions principales des ordres militaires ${ }^{54}$. À la campagne, les temps d'apogée de la richesse seigneuriale coïncident également avec d'importants travaux. Dans deux commanderies normandes, Saint-Étienne de Renneville et Sainte-Vaubourg, les

\footnotetext{
${ }^{52}$ Ce remaniement important est associé à l'installation d'une sépulture privilégiée au centre du chœur, celle du commandeur Pierre de Legé († 1269), J.-C. Bonnin, Les Templiers de la Rochelle, La Rochelle, 2000, p. 24-36; et R. Favreau, «L'épitaphe de Pierre de Legé, commandeur du Temple de La Rochelle († 1269)» Revue de la Saintonge et de l'Aunis, t. 8, 1982, p. 33-36.

53 P. Brunel, «Chronique... », Archéologie médiévale, t. 12, 1982, p. 339-40 ; t. 14, 1984, p. 330-31; t. 16, 1986, p. 202 ; et t. 17, 1987, p. 219.

${ }_{54} \mathrm{Si}$ les dimensions de l'église Saint-Rémi de Toulouse $(33 \mathrm{~m}$ x 7, $20 \mathrm{~m}) \mathrm{s}$ 'appliquent bien au statut de grand prieuré, la chapelle du Temple d'Avignon $(23,80 \mathrm{~m}$ x $8 \mathrm{~m})$ ou bien celle de l'Hôpital de Soultz ( $35 \mathrm{~m}$ x $20 \mathrm{~m}$ ) apparaissent disproportionnées pour des communautés qui devaient difficilement dépasser la dizaine de frères.
} 
Templiers arasèrent la chapelle primitive pour édifier, vraisemblablement autour de 1260-1270, une vaste église gothique ${ }^{55}$. À Lachal (com. Épinouze, Drôme), les Hospitaliers transformèrent assez rapidement en corps de logis une première chapelle romane (XII ${ }^{\mathrm{e}}$ siècle ?) qui leur avait été donnée et édifièrent un second lieu de culte, également de facture "romane» (XIII ${ }^{\mathrm{e}}$ siècle ?), qu'ils réaménagèrent profondément au $\mathrm{XV}^{\mathrm{e}}$ siècle ${ }^{56}$. À la campagne, il est possible que les frères se contentèrent pendant plus longtemps des édifices cultuels érigés lors de l'installation des commanderies. Beaucoup de chapelles conservées sont en effet de style roman et si celles-ci nous ont été léguées dans un état transformé, les remaniements ne furent souvent pas antérieurs à la fin du Moyen Âge voire à l'époque moderne. Ainsi à Montbrison (Loire), les Hospitaliers ont attendu les $\mathrm{XIV}^{\mathrm{e}}$ ou XV $\mathrm{XV}^{\mathrm{e}}$ siècles pour rebâtir le chœur de leur chapelle attribuable aux années 1170-1180 [n. 36]. Au Mayet, les frères ont longtemps jugé suffisante leur chapelle Notre-Dame et n'ont doublé cette dernière d'une chapelle Saint-Jean qu'à la fin du Moyen Âge, peut-être pour offrir un lieu de culte indépendant aux villageois ${ }^{57}$.

La fonction attachée aux édifices cultuels conditionne naturellement l'organisation des espaces. L'intérêt actuel se porte notamment sur les chapelles conventuelles qui ont acquis un statut paroissial. Comment la nécessaire division de l'espace entre la communauté régulière et les fidèles s'est-elle matérialisée ? Trouve-t-on une nef scindée en deux ou bien deux vaisseaux? Comment les circulations étaient-elles organisées ${ }^{58}$ ? Comment la chapelle paroissiale était-elle intégrée au sein de l'espace conventuel? Autant de questions qui rejoignent les analyses formulées dans le cadre des prieurés bénédictins et auxquelles les études régionales permettront bientôt de répondre clairement.

\section{La fonction funéraire}

Toute maison religieuse bénéficiant d'un lieu de culte consacré était dotée d'une aire cimétériale. En ville, le clergé séculier s'efforça souvent de limiter l'ouverture de ces cimetières aux fidèles en les réservant aux frères et aux familiers des commanderies. En milieu rural en revanche, où les ordres assumèrent un rôle

\footnotetext{
55 À moins que les chapelles primitives ne fussent enchâssées dans les édifices du XIII ${ }^{\mathrm{e}}$ s., les méthodes de reconnaissance (aérienne à Renneville, fouille de 1934-1937 à Sainte-Vaubourg) n'ayant pas permis de clarifier ce point, M. Miguet, Templiers..., op. cit., p. 313-16; et C. Naud-Lereboullet (dir.), Sainte-Vaubourg au Val de la Haye. Mille ans d'histoire. La commanderie de Sainte-Vaubourg, Veneux-les-Sablons, 1999, p. 55-63.

${ }^{56}$ Les textes comme les arguments stylistiques manquent ici pour préciser cette chronologie relative, É. Rouger, "Chronique...», Archéologie médiévale, t. 25, 1995, p. 240 ; et id., "Archéologie des élévations et archéologie funéraire. Ouverture à de nouvelles problématiques dans le grand prieuré d'Auvergne », Histoire et archéologie..., op. cit., p. 132-33.

7 L. D’Agostino, «Mayet-d'École. La commanderie Saint-Jean», Bilan scientifique de la Région Auvergne. 2003, Paris, 2004, p. 31-2 ; et id., "Chronique... », Archéologie médiévale, t. 34, 2004, p. 220.

58 À Celles et à La Racherie, la chapelle ouverte à la population était intégrée à l'enclos de la commanderie et possédait une entrée externe destinée aux fidèles mais elle était fermée du côté de l'ensemble conventuel, L. D'Agostino, «Prospection thématique... », op. cit.
} 
plus important dans l'encadrement paroissial, on peut attendre un recrutement beaucoup plus large des espaces funéraires. Le problème est donc bien souvent de déterminer le statut des zones d'ensevelissement mises au jour. Sont-elles réservées aux seuls religieux ? Sinon, quel est le degré d'ouverture aux différents cercles de laïcs gravitant autour des maisons religieuses - affiliés, familiers, protecteurs, paroissiens dépendants des commanderies ou extérieurs, pauvres, malades trépassés ? On se limitera ici à deux questions principales. Existe-t-il des profils récurrents dans le recrutement des défunts qui impliqueraient une gestion caractéristique des espaces cimétériaux ? Trouve-t-on des pratiques funéraires, si ce n'est spécifiques, du moins particulièrement représentées, dans les contextes liés aux ordres militaires?

Les églises accueillirent sans doute dès l'origine les tombes des frères, prioritairement des dignitaires, et peut-être celles de proches protecteurs laïques. Mais les commanderies étaient, de manière générale, ouvertes aux laïcs dans la vie comme dans la mort ${ }^{59}$. Il est donc logique de trouver hommes, femmes et enfants de tous âges dans leurs cimetières. Ceux-ci étaient habituellement aménagés autour des chapelles, notamment dans les commanderies rurales comme en Auvergne ${ }^{60}$. En ville, les exemples de Corbeil et de Toulouse suggèrent une hiérarchisation plus stricte des lieux d'inhumation au sein desquels l'espace ouvert à l'ensemble des fidèles est éloigné du pôle ecclésial tout en restant intégré à l'enclos conventuel. Ainsi, 1'Hôpital de Toulouse abritait trois lieux d'ensevelissement différents: l'église Saint-Rémi, le cloître et le cimetière aménagé dans l'angle sud-est de l'enclos (pl. 3) [n. 34, p. 245-59]. Il faut souligner le caractère exceptionnel de la fouille de ce dernier cimetière qui a livré plus de 1800 sépultures entre le milieu du XII ${ }^{\mathrm{e}}$ siècle et la fin du Moyen Âge ${ }^{61}$. Signalons notamment la présence d'une centaine de tombes de pèlerins qui conforte le profil charitable des Hospitaliers (ill. 5), ainsi que l'aménagement, sans doute à la fin du Moyen Âge, de caveaux contre les murs d'enceinte du cimetière évoquant assez le type du cloître-cimetière. Le recrutement devrait toutefois s'apparenter à celui d'un cimetière paroissial «classique» et il paraît plus important d'insister ici sur les espaces dont l'interprétation pose problème.

Sur la gestion des espaces funéraires, des résultats précis viennent de la fouille de Saint-Jean de Corbeil. Le cloître a fonctionné comme espace funéraire entre la fin du $\mathrm{XII}^{\mathrm{e}}$ et le milieu du $\mathrm{XIV}^{\mathrm{e}}$ siècles, parallèlement à un cimetière non

\footnotetext{
${ }^{59}$ En Provence, l'encadrement spirituel développé par les Templiers attirait de nombreuses élections de sépultures de la part des fidèles, principalement issus de la petite et moyenne aristocratie, D. Carraz, L'Ordre du Temple..., op. cit., p. 345-55.

${ }^{60}$ L. D’Agostino, «Espaces funéraires et inhumations dans les maisons de l'Hôpital de SaintJean de Jérusalem: le cas du Prieuré d'Auvergne $\left(\mathrm{XII}^{\mathrm{e}}-\mathrm{XVI}^{\mathrm{e}}\right.$ s.) », Espace ecclésial et liturgie. Actes du colloque de Nantua (24-25 octobre 2006), dir. A. Baud, N. Reveyron, à paraître.

${ }^{61}$ C'est en effet, à ma connaissance, l'unique fouille intégrale d'un «cimetière laïque » dépendant d'une commanderie urbaine. On peut espérer, malgré le ralentissement du rythme des grands aménagements urbains, que de telles découvertes se multiplieront. Une portion du cimetière, devenu paroissial au XIV ${ }^{\mathrm{e}}$ s., de la commanderie Saint-Jean de La Rochelle a ainsi été dégagée en 2006 [n. 40].
} 
fouillé, situé au sud de l'église et dont le statut était peut-être paroissial. La trentaine de sépultures mises au jour - comportant les restes de 51 individus - répond à une nette différenciation des espaces. Les galeries ont accueilli des tombes soignées, constituées de coffres anthropomorphes en plâtre - éventuellement mêlés à des éléments de pierres ou de tuiles - dans lesquels reposaient principalement des hommes habillés. Les ailes du cloître comportaient une densité importante d'inhumations caractéristique d'un espace convoité, ce dont témoigne la fréquence des réductions - jusqu'à trois dans un seul coffrage. Les sépultures étaient donc gérées comme des caveaux où les corps se sont succédé sans bousculer l'ordonnancement originel de la tombe primaire. La cour du cloître fonctionnait quant à elle comme un cimetière classique : les corps, originellement enveloppés de linceuls, reposaient dans des tombes en pleine terre. La population de la cour, fortement féminine et juvénile, était probablement constituée de laïcs proches de la commanderie - familiers, affiliés ? - tandis que les galeries devaient être réservées aux religieux [n. 16]. Cette répartition n'apparaît pas aussi clairement dans la galerie sud du cloître du grand prieuré de Toulouse où la typologie des inhumations en fosse est plus variée puisque les défunts, là encore majoritairement masculins, étaient déposés en linceul et/ou habillés dans des coffres de bois, des cercueils ou en espace colmatét $^{62}$ (ill. 6). Il est possible que la différenciation des espaces ait été moins tranchée en contexte rural ou qu'elle soit plus difficilement perceptible. Au Mayet, les sépultures des Hospitaliers inhumés en habit coexistaient avec celles d'enfants en bas âge au sein d'un même espace. Mais l'emprise des sondages paraît ici trop restreinte pour permettre de généraliser cette constatation à l'ensemble du cimetière [n. 58]. L'utilisation d'une même aire sépulcrale a également pu varier dans le temps, mais les difficultés fréquemment posées par la datation des sépultures peuvent compliquer la compréhension des phases successives d'inhumations. À L'Argentière-la-Bessée (Hautes-Alpes), la chapelle Saint-Jean de l'Hôpital installée sur une butte rocheuse a polarisé les inhumations. Au cours des XII $-\mathrm{XIII}{ }^{\mathrm{e}}$ siècles, les sépultures rupestres ou en coffrages de moellons auraient d'abord accueilli les frères, puis le recrutement se serait bientôt élargi à certains laïcs. Entre la fin du XV et le début du XVI siècles, les tombes furent ouvertes pour accueillir des ossuaires provenant du nettoyage d'un cimetière voisin, laissant entendre l'arrêt de l'inhumation des Hospitaliers ${ }^{63}$.

Il n'y a pas lieu de s'étendre ici sur les dispositions des corps tout à fait habituelles: la plupart des individus sont inhumés en décubitus dorsal, selon une orientation est-ouest, les membres supérieurs ramenés sur le thorax ou le bassin (ill. 6). Les rites funéraires observés, comme les dépôts de vases à encens (Corbeil), de pégaus (L'Argentière) ou d'une obole dans la bouche (La Salvetat), n'ont rien de particulièrement originaux non plus. Les fouilleurs de La Salvetat avaient fait quelques constatations insolites: os portant des traces de découpe et de

\footnotetext{
${ }^{62}$ L'inhumation habillée a notamment été repérée pour deux enfants, N. Pousthomis-Dalle, «Histoire... », op. cit., p. 257.

${ }^{63}$ S. Tzortzis, «L'Argentière-la-Bessée. Abords de la chapelle Saint-Jean », Bilan scientifique de la Région Provence-Alpes-Côte d'Azur. 1999, Paris, 2000, p. 35-7 ; ibid., 2001, p. 33-4; ibid., 2002, p. 43-2 ; ibid., 2006, p. 50-52 ; et id., "Chronique... », Archéologie médiévale, t. 36, 2006, p. 279.
} 
décarnisation, sépulture associée à une calotte crânienne volontaire sectionnée et à des dépôts de clous, corps désarticulé placé dans une cavité trop étroite [n. 17]. En 1977, ces pratiques avaient été mises sur le compte «de croyances et de rites attardés », mais peut-être reviendrait-on aujourd'hui sur ce type d'interprétation ? Un fait peut toutefois surprendre à la lecture des quelques études rassemblées ici : la forte représentation d'individus juvéniles. Des sépultures infantiles ont en effet été fouillées en nombre dans les maisons hospitalières d'Aphat-Ospitalia (PyrénéesAtlantiques $)^{64}$, Corbeil [n. 16], Toulouse ${ }^{65}$ et L'Argentière-la-Bessée et ont encore été repérées dans plusieurs commanderies d'Auvergne [n. 60]. Faut-il lier ces constatations à la vocation charitable des Hospitaliers qui, s'ils refusaient théoriquement l'oblation des jeunes enfants, étaient parfois tenus de recueillir les enfants abandonnés ? Ou bien la surreprésentation de ces tranches d'âges reflète-telle tout simplement la surmortalité infantile caractéristique du schéma démographique antérieur à l'ère de la vaccination ? Constatant une représentation inhabituelle de squelettes de très jeunes immatures (fœtus et nourrissons), les fouilleurs de L'Argentière ont pensé se trouver en présence d'un sanctuaire à répit ${ }^{66}$. Cette hypothèse, qui s'appliquerait assez à la dimension curative des Hospitaliers, vaudrait peut-être d'être considérée dans d'autres cas.

Les données archéologiques comme textuelles laissent encore plus mal percevoir la sélection sociale des espaces ${ }^{67}$. Quels étaient, pour les religieux ou pour leurs protecteurs laïques, les espaces ou les signes privilégiés d'inhumation? Il n'est guère étonnant que les frères aient souvent conservé jusque dans la mort les signes de leur appartenance aristocratique, à en juger par les pierres tombales, souvent de belle facture, ou par les épitaphes relevées à Soultz, Bajoles, Toulouse ou La Rochelle. Il reste encore à recenser et à étudier systématiquement ces nombreuses pièces lapidaires et leurs commanditaires. Quelle est la chronologie de leur diffusion? Étaient-elles seulement réservées aux dignitaires? Ces questions s'appliquent encore aux enfeus attestés un peu partout et dont il faudrait, là encore, entreprendre l'inventaire et les relevés systématiques ${ }^{68}$. On ne peut omettre ici les quatre enfeus adossés au mur extérieur nord de la nef de l'église Saint-Rémi de

64 «On constate une étonnante proportion d'enfants, parfois très jeunes» inhumés à l'intérieur de la chapelle de l'Hôpital de la commune de Saint-Jean-le-Vieux, A. Legaz, «Chronique...», Archéologie médiévale, t. 31, 2001, p. 424 ; et t. 33, 2003, p. 257-58.

${ }^{65}$ Sur 59 individus identifiés dans la galerie sud du cloître, 37 étaient immatures. Le cimetière ouvert aux fidèles des paroisses voisines renfermait quant à lui 400 sépultures d'enfants et d'adolescents sur un total de 1869 inhumations échelonnées entre le XII ${ }^{\mathrm{e}}$ et le milieu du XVII ${ }^{\mathrm{e}}$ s., N. Pousthomis-Dalle, «Histoire... », op. cit., p. 256-60.

${ }^{66}$ S. Tzortzis, «L'Argentière-la-Bessée... », Bilan scientifique..., op. cit., 2002, p. 43-2. Cette pratique, consistant à conduire les bébés décédés en des lieux sacrés susceptibles de les ranimer le temps d'un baptême in extremis, est en effet attestée entre le $\mathrm{XIV}^{\mathrm{e}}$ et le XIX ${ }^{\mathrm{e}} \mathrm{s}$.

${ }^{67}$ Dans le cas toulousain, les testaments et élections de sépulture n'offrent guère de précisions de localisation et les plaques funéraires ont souvent été conservées hors de leur contexte primitif, N. Pousthomis-Dalle, «Histoire... », op. cit., p. 247.

68 On en a relevé à Olloix (Puy-de-Dôme), Montbrison, Saint-Jean du Puy, Bellecombe (Isère), Lachal, Bajoles, Aphat-Ospitalia, Épailly... (pour l'Auvergne, cf. L. D'Agostino, «Espaces funéraires... », op. cit.). 
Toulouse (ill. 7). Plutôt qu'à des frères, les sarcophages étaient sans doute réservés à quelques laïcs de haut rang, mais leur utilisation ultérieure comme ossuaires et dépotoirs rend difficile la compréhension de leur fonction primaire ${ }^{69}$. La fonction funéraire des commanderies s'est en effet souvent prolongée jusqu'à une période avancée de l'Ancien Régime, notamment pour les religieux inhumés dans les églises jusqu'à la Révolution ${ }^{70}$. Les lieux d'ensevelissement ouverts aux laïcs ont longtemps fonctionné également, mais ils semblent parfois ne s'être mis en place qu'à la fin du Moyen Âge, comme à La Salvetat ou à Fontsèche ${ }^{71}$. En s'inscrivant dans la longue durée, on croisera donc avec profit les données archéologiques avec la grande variété des sources écrites - testaments, litiges sur les droits de sépulture, textes normatifs et narratifs...-, tout en élargissant la réflexion à la problématique générale de la mort chez les ordres militaires. À n'en pas douter, les frères guerriers réservent encore de belles découvertes.

Damien Carraz

Université Blaise Pascal - Clermont Ferrand 2 - EA 1001 -CHEC

\footnotetext{
${ }^{69}$ N. Pousthomis-Dalle, «Histoire...», op. cit., p. 251-56; et plus spécifiquement sur les décors : ead., «Toulouse : Hôtel Saint-Jean, ancien grand prieuré des Hospitaliers de SaintJean de Jérusalem. Décor peint des enfeus ", Bulletin monumental, t. 160, 2002, p. 189-92. Le sarcophage de l'enfeu E3, surmonté par un superbe gisant féminin, conservait quatre sujets une jeune femme, un enfant, et deux hommes âgés - dont l'inhumation a pu s'échelonner sur plusieurs siècles. Le sarcophage de l'enfeu E4, utilisé comme pourrissoir, a connu trois phases: inhumation d'un homme et deux remaniements d'ossements assimilables à des ossuaires, le premier comportant les restes d'un nourrisson, N. Pousthomis-Dalle, «Toulouse. Programme... », op. cit., p. 186-87.

${ }^{70}$ Ainsi qu'en témoignent les dalles funéraires $\left(\mathrm{XIV}^{\mathrm{e}}-\mathrm{XVIII}{ }^{\mathrm{e}} \mathrm{s}\right.$.) provenant de l'église de Soultz (P. Brunel, «Témoins de l'histoire des ordres religieux militaires en Alsace », Cahiers alsaciens d'archéologie, d'art et d'histoire, t. 33, 1990, p. 131-43), les relevés d'épitaphes $\left(\mathrm{XVI}^{\mathrm{e}}-\mathrm{XVIII}{ }^{\mathrm{e}}\right.$ s.) dans la chapelle Saint-Jean de Malte d'Avignon (D. Carraz, Une commanderie templière et sa chapelle à Avignon : étude historique et monumentale, mémoire de maîtrise, Université de Provence, 1994, vol. 1, p. 92) ou encore la présence dans l'église de l'Hôpital de Toulouse d'un grand caveau vidé en 1841 (N. Pousthomis-Dalle, «Histoire... », ibid., p. 246).

${ }^{71}$ À La Salvetat, la fonction funéraire succède à l'abandon des bâtiments de la commanderie, peut-être dès la deuxième moitié du XIV ${ }^{\mathrm{e}}$ s. [n. 17]. Utilisé entre le $\mathrm{XV}^{\mathrm{e}}$ et le XVIII ${ }^{\mathrm{e}}$ s., le cimetière de Fontsèche a livré les restes d'une centaine d'individus [n. 20].
} 


\section{ILLUSTRATIONS}

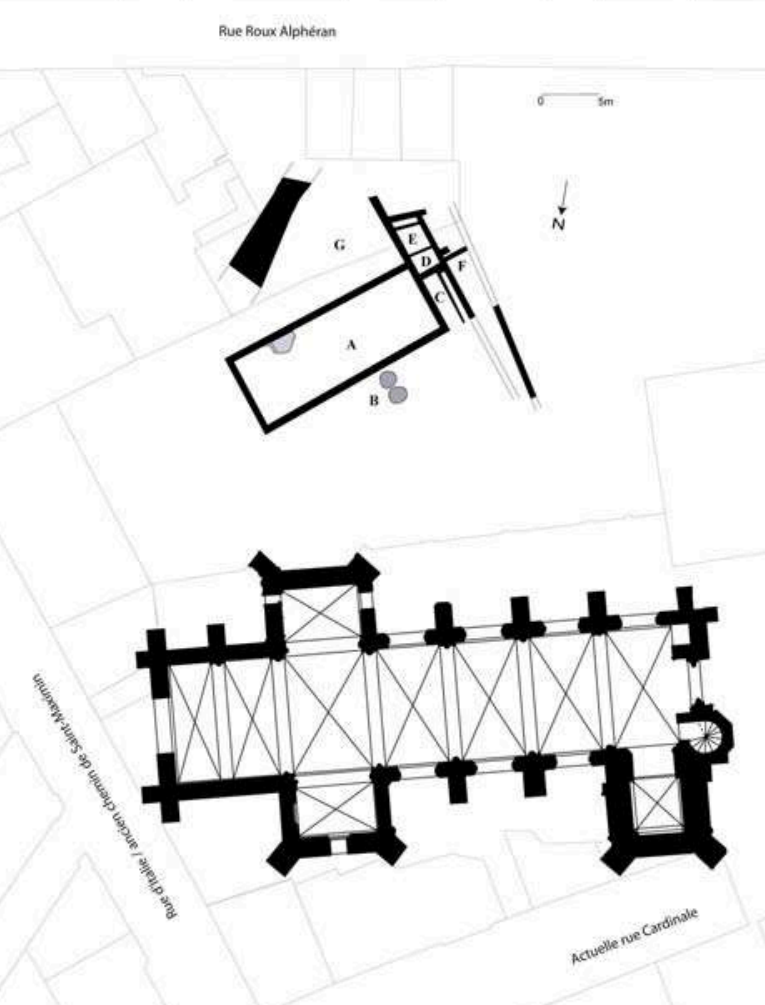

Plan 1 


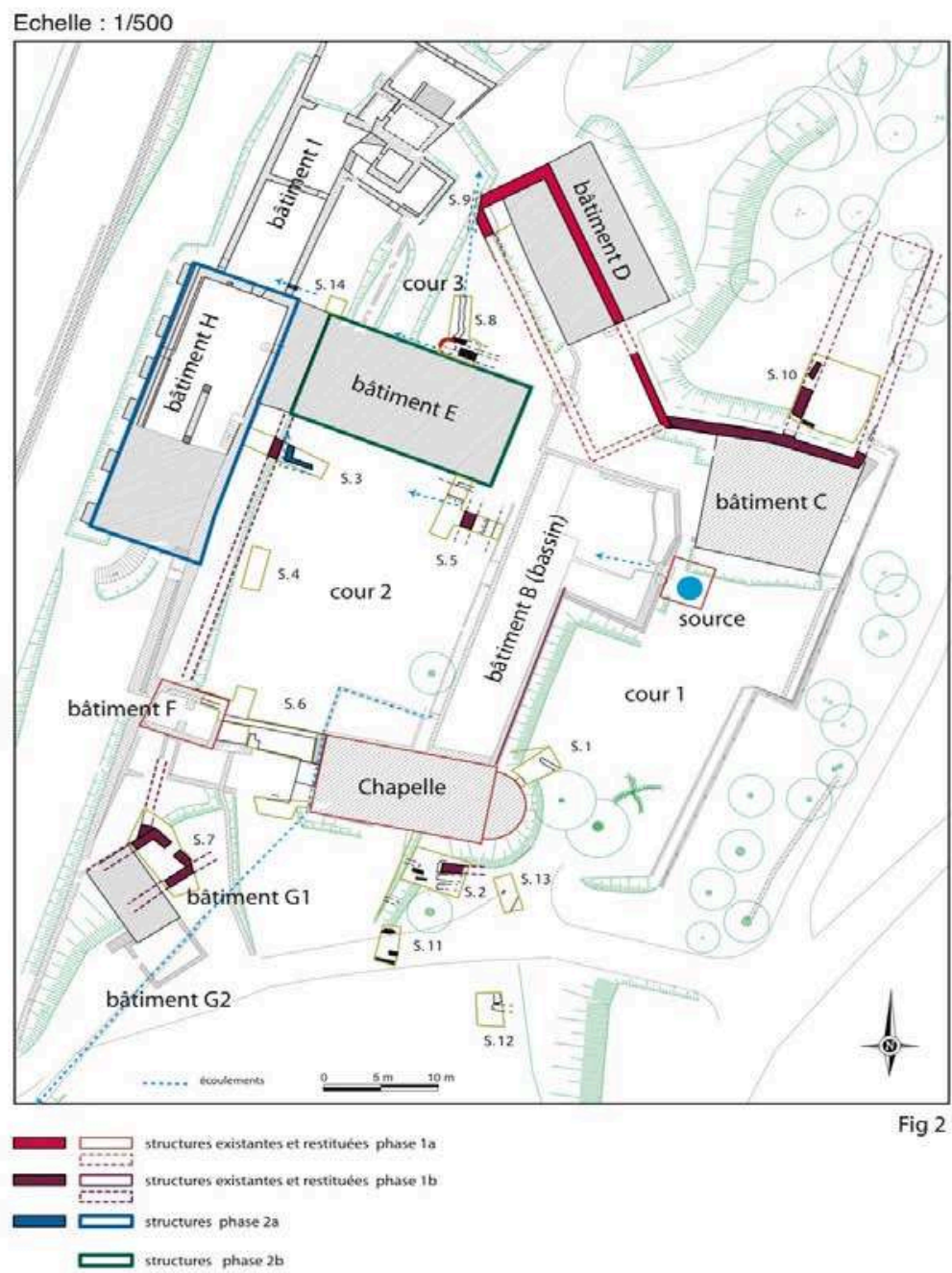

Plan 2 


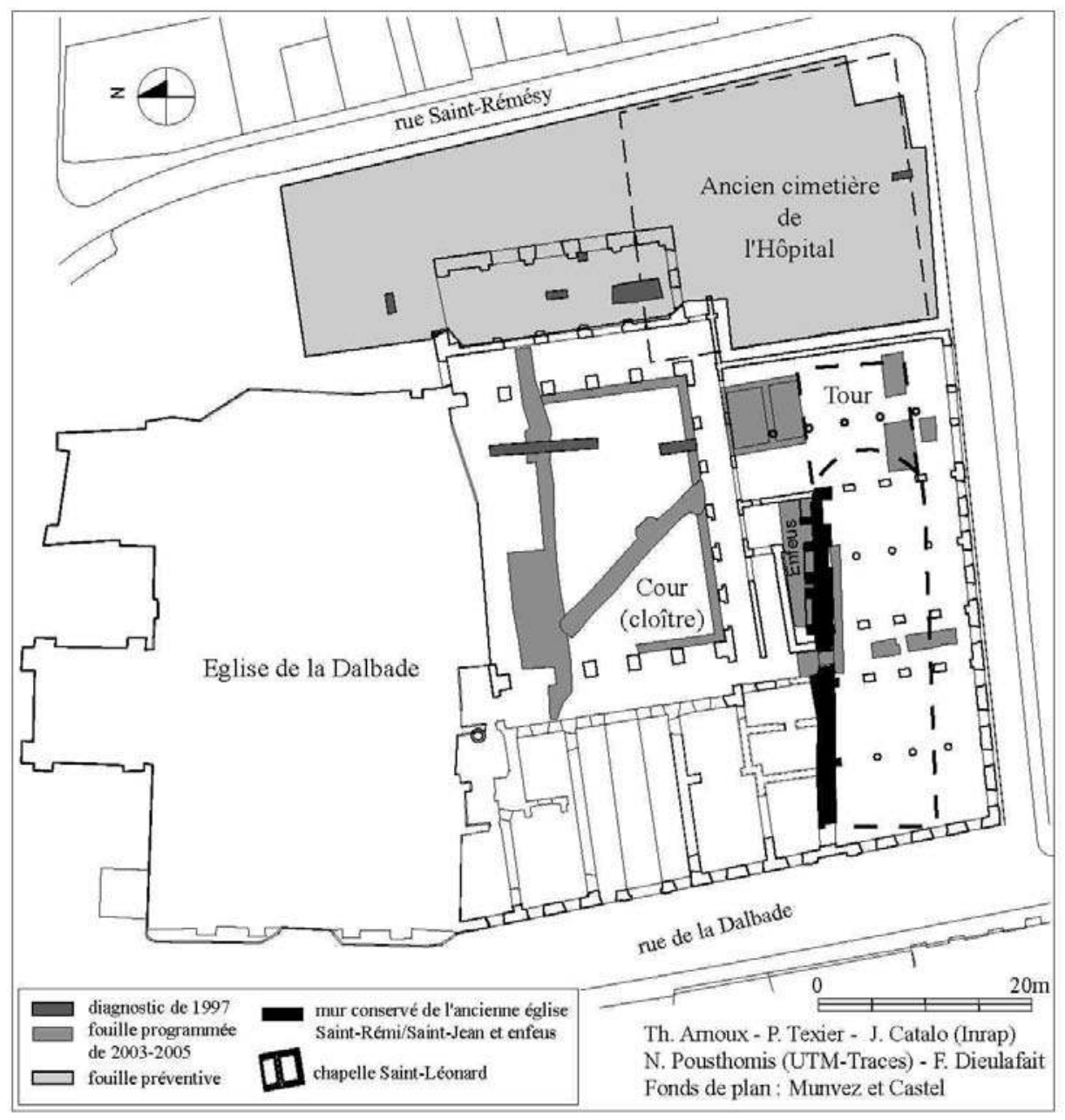

Plan 3 


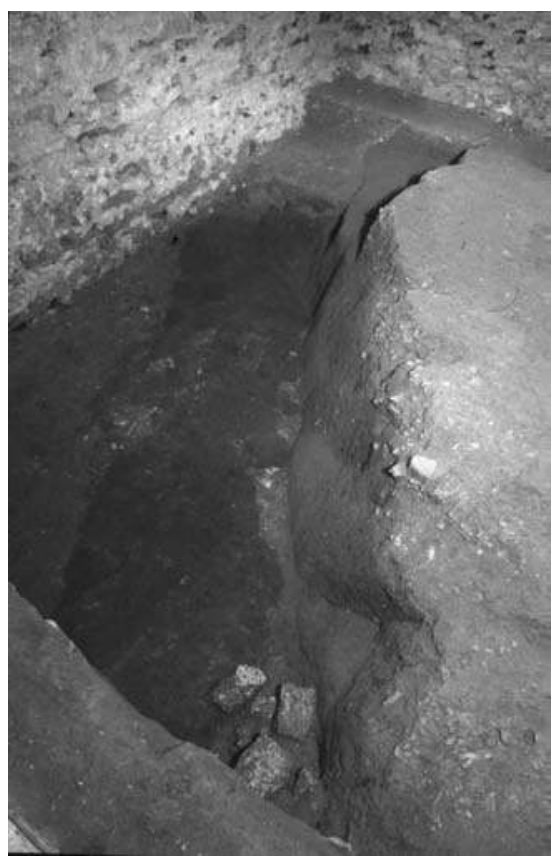

Ill. 1

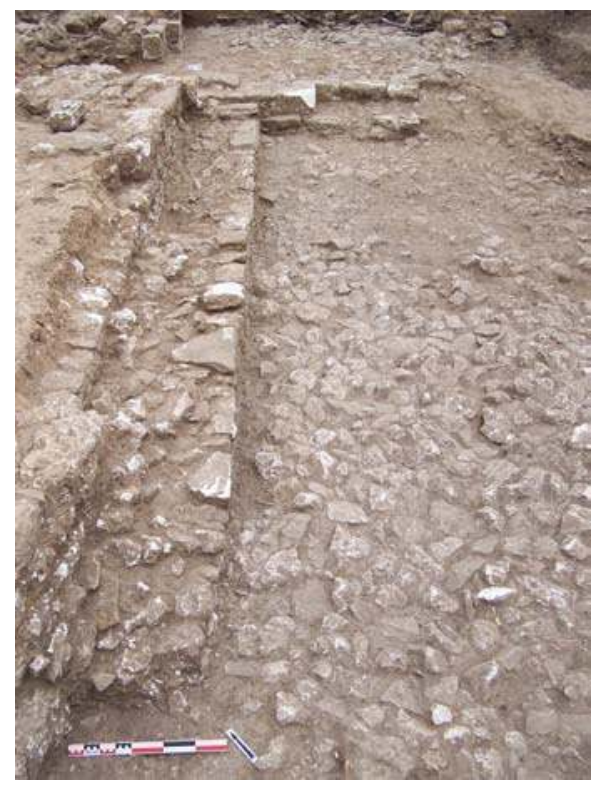

Ill. 2 


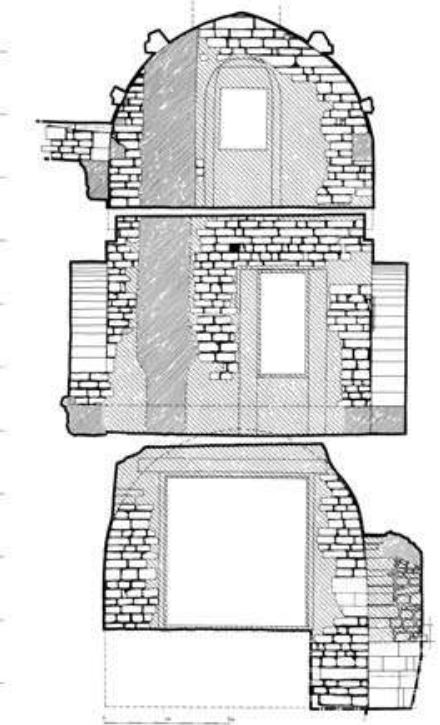

Ill 3

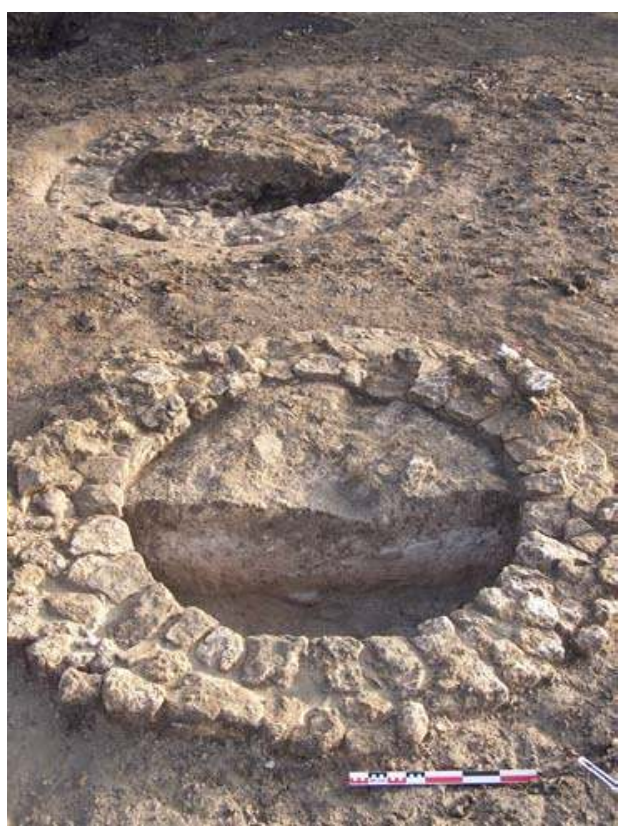

Ill 4 


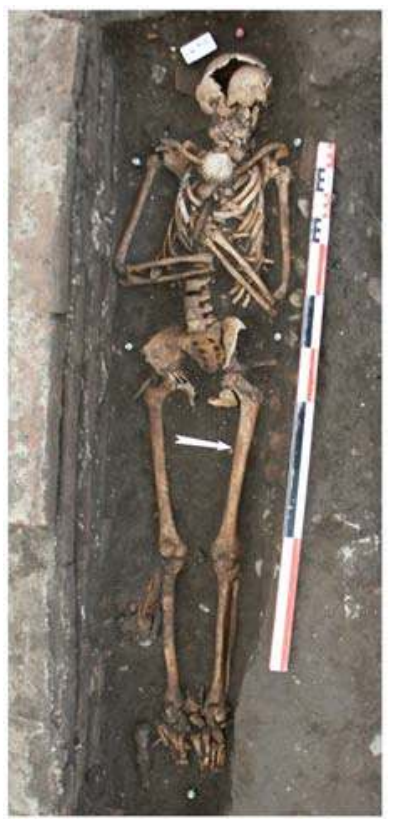

Ill 5

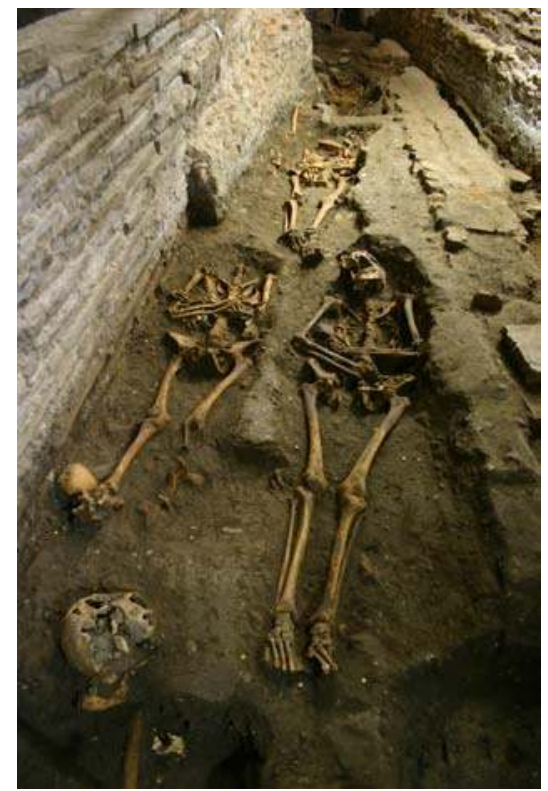

Ill 6 


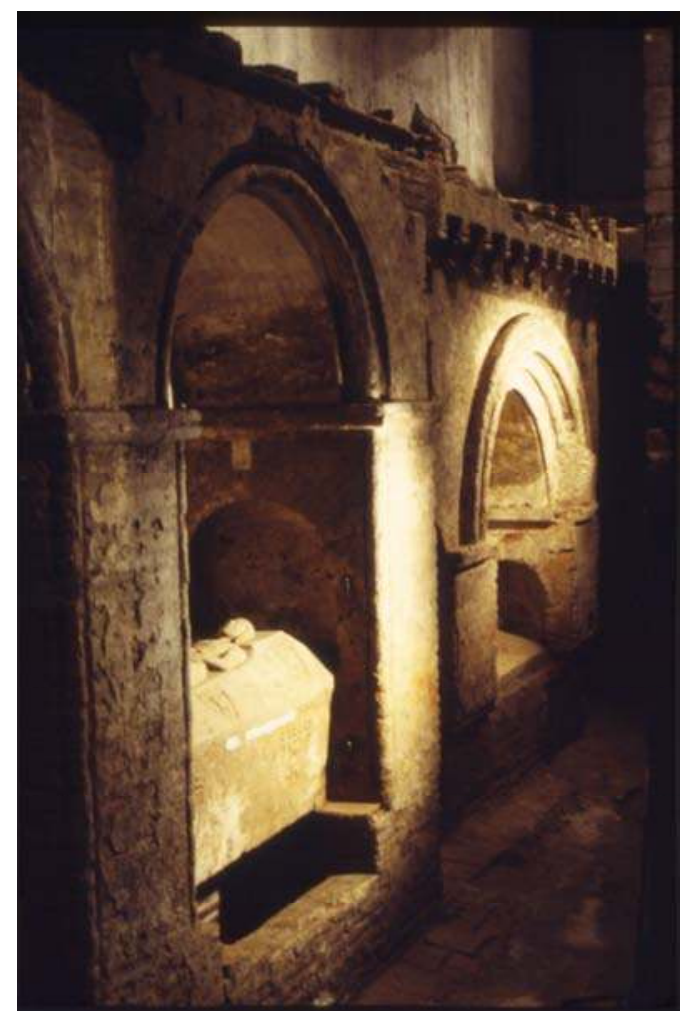

Il1 7

\section{Légendes et commentaires des illustrations}

$\underline{\text { Plans : }}$

Pl. 1 : Commanderie de l'Hôpital d'Aix (Bouches-du-Rhône) : vestige de la seconde moitié du XIII ${ }^{\mathrm{e}}$ siècle [Nuria Nin, service archéologique municipal d'Aix-enProvence, 2001]

Pl. 2 : Commanderie du Temple du Ruou (com. Villecroze, Var) [Robert Thernot, INRAP, 2004]

Pl. 3 : Grand prieuré de l'Hôpital de Toulouse : secteurs fouillés [Nelly Pousthomis et alii, UTAH-UMR 5608, 1997-2005] 


\section{Photographies :}

Ill. 1: Aix-en-Provence, commanderie de l'Hôpital (actuel Musée Granet) : excavation creusée à la fin du $\mathrm{XVI}^{\mathrm{e}} \mathrm{s}$. : négatif du rempart démantelé ou fossé de protection ? [cl. P. Reynaud].

Ill. 2: Tonnay-Charente, commanderie de Fontsèche: ce bâtiment E, qui n'a malheureusement pas livré de véritable couche d'occupation, illustre la restauration de la commanderie entre la fin du XIV et le XV ${ }^{\mathrm{e}}$ s. [cl. Patrick Ernaux, INRAP]

Ill. 3 : Richerenches (Vaucluse), maison du Temple : relevé de l'élévation est [JeanMarc Mignon, Service d'Archéologie du Département de Vaucluse, 2002]. L'analyse a permis de comprendre l'état médiéval de l'édifice débarrassé des adjonctions postérieures. La coupe montre les deux niveaux voûtés en berceau brisé ainsi que le négatif de la cheminée plaquée contre le mur est de la salle interprétée comme aula.

Ill. 4 : Tonnay-Charente, commanderie de Fontsèche : cuves maçonnées peut-être liées à une activité de tannage [cl. Patrick Ernaux, INRAP]

Ill. 5 : Toulouse, cimetière du grand prieuré : sépulture de pèlerin inhumé avec une coquille Saint-Jacques et son bourdon dont il reste l'extrémité ferrée [cl. Didier Rigal, INRAP]

Ill. 6 : Toulouse, cloître du grand prieuré : sépultures de la galerie des enfeus [cl. Jean-François Peiré, DRAC Midi-Pyrénées]

Ill. 7 : Toulouse, cloître du grand prieuré : enfeus appuyés sur le mur nord de l'église [cl. SRA Midi-Pyrénées] 